\title{
The cost of medical management of pulmonary nontuberculous mycobacterial disease in Ontario, Canada
}

\author{
A. Leber* and T.K. Marras",
}

ABSTRACT: Treatment of pulmonary nontuberculous mycobacterial (NTM) infection is complex, requiring multiple antibiotics and a prolonged treatment course. We determined the monthly cost of treating patients with pulmonary NTM infections in our clinic, a tertiary care centre in Toronto, Ontario, Canada.

We reviewed records of a single clinic at the University Health Network (Toronto) for all patients with pulmonary NTM isolates. Pharmacological and nonpharmacological treatment costs were calculated using a number of Canadian references.

172 patients were reviewed, 91 of whom were treated pharmacologically. The median total duration and cost per treated patient were 14 months (interquartile range (IQR) 9-23 months) and CAD 4,916 (IQR CAD 2,934-9,063), respectively. Median monthly drug treatment cost was CAD 321 (IQR CAD 254-458) for all patients, CAD 289 (IQR CAD 237-341) for patients receiving exclusively oral antibiotics and CAD 1,161 (IQR CAD 795-1,646) for patients whose treatment included i.v. antibiotics. The most costly oral regiment consisted of a fluroquinolone, macrolide and rifampin. In multivariable analysis, Mycobacterium abscessus infection, i.v. therapy and Mycobacterium xenopi infection were all associated with increased monthly treatment costs.

The direct medical costs of NTM infections are substantial. Less expensive alternative therapies might be most helpful for $M$. abscessus infection and when i.v. antibiotics are deemed necessary.

\section{KEYWORDS: Canada, cost-effectiveness, nontuberculous mycobacterium}

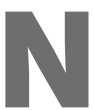
ontuberculous mycobacteria (NTM) cause pulmonary infections that can be clinically challenging at several levels. Proposed diagnostic criteria for pulmonary NTM disease are rigorous and include clinical, radiological and microbiological criteria [1]. These guidelines are meant to prevent false-positive diagnoses due to the contamination of respiratory samples with these ubiquitous organisms and the consideration of taxing therapy in patients without significant disease. The treatment is equally complex and requires a prolonged, multidrug regimen. Treatment is frequently complicated by drug intolerances to first-line agents [2] (as recommended by American Thoracic Society (ATS) guidelines [1]) especially in the elderly, which can result in chronic, continuous use of second-line antimicrobials to control the infection. Recent studies have described a significant increase in the prevalence of pulmonary NTM disease worldwide [3], making NTM an important consideration for practicing clinicians and healthcare administrators.
The long and complex therapy used in NTM pulmonary disease suggests that treatment costs are probably significant and the increasing frequency of pulmonary NTM suggests that treatment costs will have a mounting impact on the health resources. The cost of treating NTM infection is largely unknown. One study has been published, reporting a median annual treatment cost of close to CAD 20,000; however, the number of cases evaluated was relatively small and not all treatment costs were examined [4]. We sought to determine the monthly costs for the ambulatory treatment of patients with pulmonary NTM infection at a tertiary hospital clinic in Toronto, Ontario, Canada.

\section{METHODS AND MATERIALS}

A retrospective chart review was completed of all patients treated for pulmonary NTM in one clinic at the University Health Network (UHN), a large teaching hospital in Toronto. We included all patients who received a prolonged regimen

\section{AFFILIATIONS}

*Dept of Medicine, University Health Network,

\#Division of Respirology, Dept of Medicine, University Health Network and Mount Sinai Hospital, and 'Dept of Medicine, University of Toronto, Toronto, Canada.

CORRESPONDENCE

T.K. Marras

Division of Respirology

Dept of Medicine, University Health Network and Mount Sinai Hospital

Toronto Western Hospital

7E-452

399 Bathurst Street

Toronto

ON

M5T 2S8

Canada

E-mail: ted.marras@uhn.on.ca

Received:

April 082010

Accepted after revision:

Aug 242010

First published online:

Sept 032010 
( $>1$ month duration) of antimicrobial therapy, in September 2003-October 2008. Patients who did not meet ATS/Infectious Diseases Society of America (IDSA) criteria for NTM pulmonary disease were excluded. We reviewed all records in the clinic of one of the authors (T.K. Marras) to identify every eligible patient. Patients were generally treated according to ATS guidelines for pulmonary NTM disease therapy, including the allowance for the significant proportion of patients who do not tolerate intensive multidrug therapy, but may benefit from variably less intense regimens [1]. The treatment goal was not the same for all patients and did not remain the same for all patients throughout their treatment course. For some patients, the goal was to cure the infection, with a plan to treat patients for 12 months after the last positive sputum culture. Among patients for whom the initial goal of therapy was to cure the infection, some patients tolerated a prolonged course of aggressive multidrug therapy and experienced improvement, while other patients did not tolerate therapy or did not improve, so the goals of therapy were either changed to suppression of the infection or therapy was discontinued. Among patients for whom the initial goal of therapy was to suppress the infection, some patients tolerated suppressive antibiotic therapy and experienced clinical improvement, so therapy was continued, sometimes with a plan for indefinite therapy. Other patients treated with suppressive intent did not tolerate therapy or did not improve, so therapy was discontinued. We have found that this approach to pulmonary NTM disease is appropriate in our setting and report on the cost of treating patients using this flexible, inclusive approach.

Medication costs were calculated to estimate the total number of doses of each drug that was used. All clinic records were reviewed to determine the drugs and doses that patients were taking during each month of treatment. Oral antibiotic costs included dispensing fees, and were calculated based on Ontario retail costs provided by a large Canadian retail pharmacy chain. We rarely used rifabutin, reflecting the relatively high toxicity compared with rifampin, as highlighted by the latest ATS guidelines [1]; therefore, none of the patients in this series was treated with rifabutin. Clofazimine (Lamprene; Novartis Pharmaceuticals Corporation, East Hanover, NJ, USA) is not commercially available in Canada. It is currently being provided free of charge on compassionate grounds, through Health Canada's Special Access Program, by its manufacturer. The cost of clofazimine was not made available to us and is, therefore, not included in our calculations. Parenteral antibiotic acquisition costs and the associated nursing fees for home administration were determined using a Toronto area homecare programme and their contracted pharmacy. Costs of central line insertion and associated infusion costs were also designated as drug costs.

Nonmedication costs included physicians' fees, medical facility fees and patient monitoring. Physicians' fees, including the pulmonary specialist, radiologists and interventional radiologists, were calculated using the schedule of benefits from the Ontario Ministry of Health and Long Term Care and counting every applicable patient encounter. Thoracic computed tomography (CT) scans were routinely performed initially, after 4-6 months of therapy, at the time of significant clinical deterioration and at the end of therapy. Chest radiographs were used predominantly in the setting of acute changes. All of these radiographic costs were included. In-person (reimbursed) pulmonology assessments were generally performed every 3 months. Costs incurred by the hospital for clinic visits, infusion centre administration of antibiotics, radiological tests and interventional procedures were calculated using the UHN "case costing" database for every applicable patient encounter. The costs of monitoring patients on therapy, according to costs for monthly complete blood counts, and aspartate transaminase, alanine transaminase and total bilirubin levels (for patients receiving a rifamycin), monthly sputum acid-fast staining and mycobacterial culture, were also designated as nondrug costs. We studied medical treatment costs, so the costs of initial diagnostic investigations were not included in the analysis. The costs of baseline investigations at the initiation of therapy were, however, included as part of our analysis. A small proportion of our patients had surgical therapy for their NTM disease. The costs of this intervention were not available to us and so are not included.

All costs were calculated according to 2008 rates, and are presented in Canadian dollars and summarised rounded to the nearest dollar. Given the variable treatment length for pulmonary NTM, the monthly treatment cost was the focus of study, and was calculated as the total treatment cost during the observation period divided by the total number of treatment months. Total treatment costs are also presented but do not include only completed courses of intensive "curative" therapy. We included data for all patients who were treated during the study period, regardless of whether they had completed therapy with curative intent, discontinued potentially curative therapy because of toxicity or futility, utilised ongoing suppressive therapy or discontinued suppressive therapy because of toxicity or futility.

Bivariate comparisons for categoric variables were made with continuity-corrected Chi-squared tests or Fisher's exact tests as appropriate. Bivariate comparisons for cost (which was not normally distributed) were made with Mann-Whitney U-tests. After assessment for collinearity, multiple linear regression, using backward model selection, was used to model logtransformed monthly treatment cost (outcome variable), using sex, age, presence of cavitation on $\mathrm{CT}$, acid-fast stain status, use of i.v. therapy and NTM species as predictor variables. Data were entered into an electronic database (Access 2000; Microsoft, Redmond, WA, USA) and were analysed using statistical software (SAS 8.02; SAS Institute, Cary, NC, USA). This study was approved by our institutional review board, without requiring informed consent.

\section{RESULTS}

A total of 172 patients, seen at the UHN respiratory clinic for pulmonary NTM infection in 2003-2008, were reviewed. 91 patients were followed for $\geqslant 1$ month and treated with prolonged antimicrobial therapy and, therefore, included in subsequent analysis. Baseline characteristics of the 91 patients are presented in table 1 . The majority of patients were thin, elderly females, consistent with previously reported results [1]. Physician-defined pre-existing chronic obstructive pulmonary disease (COPD) was present in $\sim 40 \%$ of cases; however, obstructive airway disease, as defined by pulmonary function testing, was present in a much higher proportion. This discrepancy could be due to the direct effect of NTM infection 


\begin{tabular}{|c|c|c|c|}
\hline \multirow{2}{*}{$\begin{array}{l}\text { TABLE } 1 \\
\text { Characteristic }\end{array}$} & \multicolumn{3}{|c|}{$\begin{array}{l}\text { udy subjects with pulmonary } \\
\text { cobacterial (NTM) disease }\end{array}$} \\
\hline & Total & Males & Females \\
\hline Subjects $\mathbf{n}$ & 91 & 31 & 60 \\
\hline Age yrs & $61.7 \pm 13.6$ & $62.3 \pm 16.0$ & $61.5 \pm 12.4$ \\
\hline \multicolumn{4}{|l|}{ Concomitant lung disease } \\
\hline COPD & $21(23)$ & $9(29)$ & $12(20)$ \\
\hline Asthma & $16(18)$ & $9(29)$ & $7(12)$ \\
\hline Interstitial lung disease & $5(5)$ & $4(13)$ & $1(2)$ \\
\hline \multicolumn{4}{|l|}{ Previous NTM infection } \\
\hline Previous diagnosis & $28(31)$ & $5(16)$ & $23(38)$ \\
\hline Previous diagnosis and treatment & $23(25)$ & $4(13)$ & $19(32)$ \\
\hline $\mathrm{BMI} \mathbf{k g} \cdot \mathrm{m}^{-2}$ & $22.2 \pm 4.5$ & $23.0 \pm 4.1$ & $21.9 \pm 4.7$ \\
\hline \multicolumn{4}{|l|}{ Pulmonary function tests } \\
\hline Obstructive ${ }^{\#}$ & $55(60)$ & $22(71)$ & $33(55)$ \\
\hline Restrictive & $10(11)$ & $4(13)$ & $6(10)$ \\
\hline Impaired diffusion capacity ${ }^{+}$ & $49(54)$ & $21(68)$ & $28(47)$ \\
\hline \multicolumn{4}{|l|}{ Clinical manifestations of NTM } \\
\hline Constitutional symptoms $s^{\S}$ & $50(55)$ & $18(58)$ & $32(53)$ \\
\hline Cough & $82(90)$ & $26(84)$ & $56(93)$ \\
\hline Dyspnoea & $33(36)$ & $12(39)$ & $21(35)$ \\
\hline Haemoptysis & $29(32)$ & $8(26)$ & $21(35)$ \\
\hline Chest pain & $6(7)$ & $2(6)$ & $4(7)$ \\
\hline \multicolumn{4}{|l|}{ Radiological manifestations of NTM } \\
\hline Multifocal bronchiectasis & $60(66)$ & $14(45)$ & $46(77)$ \\
\hline Multiple nodules & $75(82)$ & $22(71)$ & $53(88)$ \\
\hline Cavity & $25(27)$ & $9(29)$ & $16(27)$ \\
\hline \multicolumn{4}{|l|}{$\begin{array}{l}\text { Microbiological manifestations } \\
\text { of NTM }\end{array}$} \\
\hline 3 positive sputum cultures ${ }^{f}$ & $53(58)$ & $14(45)$ & $39(65)$ \\
\hline Bronchial wash and positive culture & $36(40)$ & $17(55)$ & $19(32)$ \\
\hline Surgical biopsy/resection & $2(2)$ & $0(0)$ & $2(3)$ \\
\hline Acid-fast stain positive & $55(60)$ & $19(63)$ & $36(59)$ \\
\hline \multicolumn{4}{|l|}{ NTM speciation ${ }^{\# \#}$} \\
\hline MAC $\%$ & $74(81)$ & $22(71)$ & $52(87)$ \\
\hline Mycobacterium xenopi & $17(19)$ & $7(12)$ & $10(17)$ \\
\hline Mycobacterium abscessus & $6(7)$ & $2(6)$ & $4(7)$ \\
\hline Mycobacterium fortuitum & $3(3)$ & $1(3)$ & $2(3)$ \\
\hline Mycobacterium gordonae & $3(3)$ & $1(3)$ & $2(3)$ \\
\hline Mycobacterium kansasii & $1(1)$ & $1(3)$ & $0(0)$ \\
\hline
\end{tabular}

Data are presented as mean \pm SD or $\mathrm{n}(\%)$, unless otherwise stated. COPD: chronic obstructive pulmonary disease; BMI: body mass index; MAC: Mycobacterium avium complex. ": forced expiratory volume in $1 \mathrm{~s}(\mathrm{FEV} 1) /$ forced vital capacity $(\mathrm{FVC})<0.7{ }^{\circ}: \mathrm{FEV} 1 / \mathrm{FVC}>0.7$ and total lung capacity $<80 \%$ predicted. ${ }^{+}$: diffusion capacity $<75 \%$ pred. ${ }^{\text {s: }}$ : fever, weight loss, malaise and fatigue. ${ }^{f}$ : three positive sputa were required according to the prior version of American Thoracic Society NTM guideline (2003) [5], contemporary with treatment of most patients in the cohort; this criterion differs from current (2007)

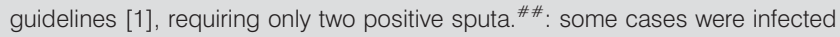
with more than one organism. " ". avium or Mycobacterium intracellulare.

on the airways, the development of bronchiectasis or a preexisting but undetected abnormality of the airways that predisposes patients to pulmonary NTM infection.

All patients met ATS criteria for pulmonary NTM disease. The patients' clinical, radiological and microbiological features are illustrated in table 1. Radiologic nodular bronchiectasis was two- to three-fold more common than cavitation. The majority of patients were infected with NTM of the Mycobacterium avium complex (MAC) (70\%), followed by Mycobacterium xenopi $(17 \%)$ and others $(11 \%)$. The median duration of treatment was 14 months (interquartile range (IQR) 9-23 months). Median treatment duration was not significantly different between MAC and M. xenopi (15.5 versus 12 months; $\mathrm{p}=0.06$ ). The small number of patients infected with other species of NTM limited further comparisons. Cavitation on CT was not significantly associated with the duration of treatment in bivariate analysis (14 months with cavitation versus 12 months without cavitation; $\mathrm{p}=0.66$ ).

The majority of patients were treated with one out of six different oral antibiotic regimens, the choice of which was largely determined by patient tolerability and medication toxicities. i.v. therapy usually comprised amikacin, which was used in 23 cases. The individual costs of antibiotics and nonmedication treatment component costs are presented in the online supplement. Daily drug costs, for average doses of the commonly used medications, were as follows. Macrolides: CAD 2.77-4.94 (varies by agent); ethambutol: CAD 0.62; rifampin: CAD 2.48; fluoroquinolones: CAD 2.82-6.21 (varies by agent). Two patients with Mycobacterium abscessus received 3 months of carbepenem therapy (approximate wholesale cost of CAD 50 per dose) in conjunction with i.v. amikacin. This cost was not included in the analysis. No other i.v. antibiotics were used. The frequency of commonly prescribed antibiotic regimens is shown in figure 1a. The median monthly cost of common antibiotic regimens is shown in figure $1 \mathrm{~b}$. Clofazimine was used in 18 patients; however, it was not included in cost calculations, as it is made available free of charge and cost information was not made available to us. There was no significant difference in cost between the different oral antibiotic regimens. A significant increase in cost occurred with i.v. amikacin.

The median monthly and total medication and nonmedication costs are shown in table 2. Greater than two-thirds of the cost was incurred from medications. Table 3 highlights cost differences between patients treated with exclusively oral therapy and patients treated with parenteral plus oral therapy. The difference in cost of nearly CAD 900 per month was driven almost exclusively by the increase in drug acquisition and administration costs. In multivariable analysis, total monthly cost was significantly associated only with infection with M. abscessus, use of i.v. antibiotics and infection with M. xenopi. Multivariable model results and interpretation are summarised in table 4.

\section{DISCUSSION}

In our study of the treatment cost of pulmonary NTM infections, we observed an average monthly cost of approximately CAD 500. Drug costs were responsible for $\sim 70 \%$ of the total treatment cost. As expected, treatment costs rose dramatically with the use of $i . v$. antibiotics and in the presence of $M$. abscessus, two variables that were often, but not always, associated (i.v. therapy was invariably used for M. abscessus but also for numerous patients with other NTM species). In multivariable modelling, parenteral therapy added $\sim \mathrm{CAD}$ 700 to the monthly treatment cost, independent of other 

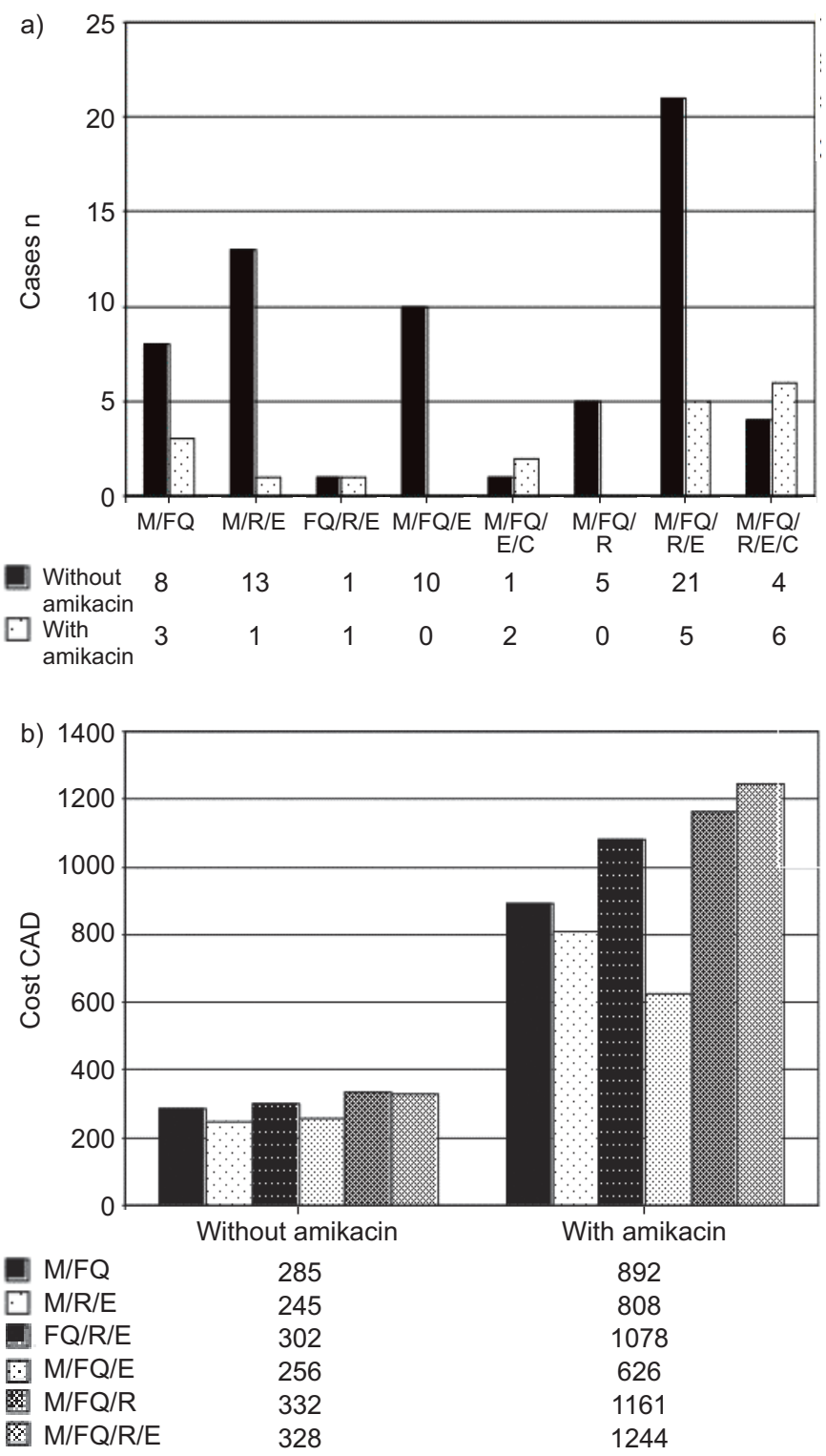

FIGURE 1. a) Frequency of commonly prescribed antibiotic regimens. 10 cases were treated with other antibiotic regimens. b) Monthly cost of common antibiotic regimens (drug acquisition and administration costs only). M: macrolide; FQ: fluoroquinone; R: rifampin; E: ethambutol; C: clofazimine.

variables. Additional results from multivariable modelling included finding that $M$. xenopi was associated with greater treatment costs than MAC, but there was no clear cost association with cavitation on CT scan, age or sex.

A recently published retrospective cohort study [4] examining the medication costs associated with the treatment of 25 cases of pulmonary NTM infection in the USA found monthly and annual medication costs $44 \%$ and $15 \%$ higher, respectively, compared with our study. There are several factors that probably contributed to the large cost difference. The prior study had a higher prevalence of M. abscessus $(22 \%$, compared with $7 \%$ in the present study), an organism that is associated with a high treatment cost. In addition, the median number of antimicrobials employed in the prior study was five, compared

\begin{tabular}{|c|c|c|c|c|}
\hline \multirow{2}{*}{$\begin{array}{l}\text { TABLE } 2 \\
\text { Cost }\end{array}$} & \multicolumn{4}{|c|}{$\begin{array}{l}\text { Total and monthly medication and detailed } \\
\text { nonmedication treatment costs of pulmonary } \\
\text { nontuberculous mycobacterial infection }\end{array}$} \\
\hline & & $\begin{array}{c}\text { Patients } \\
\text { n }\end{array}$ & $\begin{array}{l}\text { Total cost } \\
\text { CAD }\end{array}$ & $\begin{array}{c}\text { Monthly cost } \\
\text { CAD }\end{array}$ \\
\hline \multicolumn{5}{|l|}{ Medication } \\
\hline All patient & & 91 & 4916 (2934-9063) & 321 (254-458) \\
\hline i.v. and or & antibiotics & 23 & $20143(9451-31109)$ & $1161(795-1646)$ \\
\hline Oral antib & cs only & 68 & 3603 (2306-5436) & 289 (237-341) \\
\hline \multicolumn{5}{|c|}{ Nonmedication } \\
\hline All patient & & 91 & $2029(1461-2667)$ & $144(99-204)$ \\
\hline Physicia & visits & & 865 (496-1269) & $57(38-101)$ \\
\hline Radiolo & & & $585(390-780)$ & $33(23-55)$ \\
\hline Sputum & sting $^{\#}$ & & $263(106-536)$ & $21(10-42)$ \\
\hline Drug toxic & monitoring" & & $144(12-366)$ & $12(1-12)$ \\
\hline Grand total & & & 6694 (4460-11761) & $499(387-711)$ \\
\hline
\end{tabular}

Data are presented as median (interquartile range), unless otherwise stated

\#: smear and culture. $`$ : blood tests and audiograms.

with only three in our study, and we did not include the treatment cost of clofazimine in 18 patients. Finally, the drug costs were much higher in that study. The monthly cost of a standard first-line regimen comprising a macrolide, rifampin and ethambutol was $\sim$ USD 470 in that study, compared with CAD 245 in our study. The present study also differed from the prior study in that we included all readily quantifiable treatment costs, rather than focusing exclusively on drug acquisition costs. We think that our study provides a reasonable estimate of the total costs of treating pulmonary NTM infection in Ontario.

Particularly relevant to the overall financial burden of treating pulmonary NTM disease in a population, is choosing the appropriate patients to treat. In our NTM database, only 91 of the 172 patients were actively treated with antibiotic therapy. It is well recognised that making the distinction between pulmonary "colonisation" and "disease" is not always easy, and has been addressed through the creation of explicit diagnostic guidelines. Despite these guidelines, the decision to treat patients with pulmonary NTM disease remains difficult, even for experts in the field [1]. Therefore, we feel that obtaining the expertise of specialised physicians during the course of diagnosis and/or treatment would better identify those who required treatment, and could have a substantial impact on the overall disease cost.

In considering whether the financial cost of a medical treatment is acceptable, it can be helpful to compare it with other accepted therapies. Pulmonary NTM infections are probably less expensive to treat than other treatment-resistant infections, such as multidrug-resistant tuberculosis (MDR-TB), or chronic infections, such as HIV. The median outpatient costs of treating MDR-TB in seven HIV-seronegative patients in San Francisco, CA, USA was USD 21,929, over a mean duration of therapy of 98 weeks [6]. US AIDS surveillance data in 20022003 estimated the annual average cost of antiretroviral therapy was USD 12,665 per patient [7]. 
TABLE 3 Treatment costs for pulmonary nontuberculous mycobacterial infection by use of parenteral therapy

\begin{tabular}{ccc} 
Patients & p-value ${ }^{\#}$ \\
\hline All $\quad$ Oral therapy only $\quad$ Combined parenteral and oral therapy
\end{tabular}

Patients n
Cost CAD
Medication
Monthly
Total
Nonmedication
Monthly
Total
Total
Monthly
Total

91

68

23

$289(237-341)$
$3603(2306-5436)$
$132(93-184)$
$1915(1324-2308)$
$423(357-538)$
$5388(4088-7584)$

$1161(795-1646)$
$20143(9451-31109)$
$192(148-222)$
$2642(2080-4217)$
$1300(989-1813)$
$23022(11761-33791)$

$<0.0001$

$<0.0001$

4916 (2934-9063)

$144(99-204)$

$2029(1461-2667)$

$499(387-711))$

$6694(4460-11761$

23022 (11761-33791)

0.01

$<0.0001$

Data are presented as median (interquartile range), unless otherwise stated. ${ }^{*}$ : for difference between patients treated with oral therapy only versus parenteral plus oral therapy.

We feel the cost of treating pulmonary NTM disease is closer in cost to the outpatient treatment of more common chronic diseases, such as diabetes mellitus and congestive heart failure. In a large cross-sectional study published by the American Diabetes Association in 2007, the annual per capita expenditure for outpatient care (not including emergency room visits) for individuals with diabetes who were $>65$ yrs of age was USD 3,319 [8]. In 2004, the average annual cost of outpatient treatment of $>1,500$ patients with congestive heart failure was USD 3,837 [9]. We estimated the drug cost of treating COPD, type 2 diabetes mellitus and symptomatic coronary artery disease using common drug combinations and doses. In Ontario, the approximate monthly drug costs for treating COPD (using fluticasone/salmeterol, tiotropium and salbutamol) is CAD 323, symptomatic coronary artery disease (using a $\beta$-blocker, acetylsalicylic acid, statin and an angiotensin-converting enzyme (ACE) inhibitor) is CAD 196, and type 2 diabetes mellitus (using a biguanide, sulfonylurea, thiazolidinedione, statin, ACE inhibitor and acetylsalicylic acid) is CAD 319. In a similar cost range, we observed that a standard first-line regimen for treating pulmonary NTM infection (using a macrolide, rifampin and ethambutol) was CAD 245 per month. Although data regarding objective benefits in treating pulmonary NTM disease are not very well established, the cost of treatment does not appear to be inconsistent with accepted costs of ongoing treatment of common chronic diseases.

We speculate that the use of "guideline therapy", namely a macrolide, rifampin and ethambutol, as first-line treatment is the most cost-effective approach to the treatment of NTM disease in Canada. Assuming an 18-month course of treatment, the cost savings (based on our observed costs) of guideline therapy per treatment course versus other regimens, such as floroquinolone/rifampin/ethambutol, macrolide/fluroquinolone/ethambutol or fluroquinolone/rifampin/macrolide, is CAD 1,026, CAD 198 and CAD 1,566, respectively. Obviously the effectiveness of the regimen must be considered when considering cost saving approaches. However, at present, there is a paucity of studies that compare the effectiveness of different regimens. JENKINS et al. [10] compared the efficacy of a 2-yr treatment course of clarithromycin, rifampin and ethambutol versus ciprofloxacin, rifampin and ethambutol. There was

\section{TABLE 4 Results of multiple linear regression for total monthly treatment cost}

\begin{tabular}{lccc} 
Variable & Parameter estimate & Final p-value & $\begin{array}{c}\text { Approximate associated increase in total } \\
\text { monthly treatment cost CAD }\end{array}$ \\
\hline Mycobacterium abscessus & 1.27 & $<0.0001$ & $2700^{\#}$ \\
i.v. therapy & 0.90 & $<0.0001$ & $700^{\circ}$ \\
Mycobacterium xenopi & 0.28 & 0.0325 & $250^{+}$ \\
Intercept & 5.69 & $<0.0001$ & Not applicable \\
\hline
\end{tabular}

Multiple linear regression using backward model selection, modelling log-transformed monthly treatment cost (outcome variable), using sex, age, presence of cavitation on computed tomography, acid-fast stain status, use of $i . v$. therapy and nontuberculous mycobacterial species as predictor variables. Variables that remained in the model with a $p$-value of $<0.05$ are shown. Model $r^{2}=0.71$. * : presence of $M$. abscessus compared with other organisms. ": addition of $i . v$. therapy in cases of Mycobacterium avium complex (MAC) or M. xenopi. ${ }^{+}$: presence of $M$. xenopi compared with MAC 
no statistically significant difference between the two regimens in their primary end-points of death due to NTM and treatment failure (defined as positive sputum cultures on two separate occasions during the previous 3 months of treatment). As a secondary end-point, JENKINS et al. [10] compared the tolerability of these regimens and again found no statistically significant difference in the two regimens. Accepting the results from this complex study at face value, we would conclude that the combination of a macrolide, rifampin and ethambutol is the most cost-effective regimen due to its lower price, effectiveness and tolerability profile. It is even more difficult to address the cost-effectiveness of amikacin (or another aminoglycoside), the major factor driving large increases in cost of therapy. Guidelines recommend considering the use of injectable aminoglycosides in the initial therapy of cavitary MAC disease and advise their use in severe, advanced or recurrent disease. However, controlled data regarding the effectiveness of aminoglycosides in pulmonary NTM are limited. КовАSHI et al. [11] randomised patients with pulmonary MAC to clarithromycin/rifampin/ethambutol plus either streptomycin or placebo injections. Patients in the streptomycin group did slightly better, although the differences were not statistically significant. Streptomycin appeared to be particularly beneficial (for clinical and microbiological outcomes) among patients with radiographically extensive disease. It appears that an injected aminoglycoside is beneficial in extensive pulmonary MAC, but there are inadequate data to assess its cost-effectiveness.

It is interesting to note that a minority of patients were receiving the first-line recommended therapy for MAC (macrolide/rifampin/ethambutol). This observation was present despite our general practice to introduce a three-drug first-line regimen when possible. Even when including regimens that additionally contained amikacin or a fluoroquinolone, the total number of patients with these regimens comprised only $44 \%$. There are probably several reasons for this observation. Although we did not formally study regimen tolerability, we think that a siginificant proportion of patients had been intolerant of rifampin, explaining the substantial proportion of patients using nonrifampin-containing regimens. Furthermore, a very small proportion could not tolerate ethambutol, usually due to ocular toxicity. Also, patients with M. abscessus were not generally prescribed rifampin, due to the resistance of the isolates, and often received carbapenem therapy. Finally, many of our patients had recurrent or difficult-to-treat disease that led to the addition of more drugs, including fluoroquinolones, amikacin and clofazimine. Our regimen choice is, therefore, reflective of the spectrum of NTM species treated in our clinic and possibly drug intolerance that is common in treating pulmonary NTM [2].

The frequency of pulmonary NTM has been increasing substantially in Ontario [3], suggesting that the cost of treatment will have a mounting impact on the health resources, underscoring the relevance of studies like the present investigation. We think that our results are generally applicable in Canada and, with some modification for differences in healthcare costs, in other jurisdictions as well. Our study is the largest and most comprehensive investigation of the cost of treating pulmonary NTM disease to date, including drug acquisition and administration costs, as well as physician, facility and testing fees (audiology, biochemistry, haematology, microbiology and radiology). Our patients are probably representative of other populations of pulmonary NTM patients, as we ensured that all cases met the ATS diagnostic criteria for NTM disease. The drug regimens were also generally in close accordance with ATS/IDSA guidelines, although fluoroquinolones, drugs not recommended in firstline regimens, were used extensively. The fraction of patients in our clinic who were treated (53\%) is probably in accordance with the observation that pulmonary NTM disease, even in the presence of significant symptoms, radiographic abnormalities and microbiologic evidence of disease, may be relatively indolent and progress only very slowly. Patients were offered antimycobacterial drug treatment if the clinical opinion was such that the benefits of therapy were likely to outweigh the toxicities. Generally, patients were not treated if they had relatively mild and nonprogressive symptoms, and nonprogressive lesions on serial chest imaging.

Although our study offers the first comprehensive analysis of the cost of treating pulmonary NTM, there are several limitations. We did not have the cost of clofazimine available to us, so this cost was omitted from our analysis. The impact of these missing data is probably small, however, since $<20 \%$ of our patients were treated with this drug. We also did not include the cost of treatment periods with inhaled amikacin, since this therapy is less commonly used and we had few patients who received it. The latter would likely make our costs estimates thereof unreliable. Because only three patients received inhaled amikacin during the study period, it is unlikely that omitting the periods of inhaled amikacin greatly affected our results. Although we did not determine the costs surrounding the use of inhaled amikacin, we think its use would be less than for i.v. (assuming similar doses), despite the costs of an air compressor, saline and nebulisers, since inhaled therapy eliminates nursing costs and, in our practice, reduces biochemical and audiographic monitoring for toxicity. Also, if amikacin is used exclusively by inhalation, costs of central line insertion are avoided. We did not include the cost of the adverse effects of treatment. The multidrug regimen and prolonged course of therapy of pulmonary NTM is well known for high rates of adverse reactions [2]. BALLARINO et al. [4] recorded the frequency of adverse drug reactions ranged from $18 \%$ with azithromycin to $75 \%$ with levofloxacin. Adverse drug reactions may lead to more clinic visits, additional diagnostic tests and the use of more expensive, second-line agents, but this cost would have been captured using our methodology. In our cohort, most toxicities were managed by telephone, the costs of which are not included, since this is not a reimbursable service in our health system. One of our patients developed rifampin-related systemic illness, diffuse petichiae and ecchymoses with severe thrombocytopenia, for which hospitalisation was required. The in-patient costs of this toxicity were not available to us and not included in our analysis Although the in-patient cost of pulmonary NTM disease was also not explored, hospitalisation for initiation or modification of therapy did not occur in patients, outside of the unusual situation of pulmonary resection for localised disease, an intervention that is used in the minority of patients. The majority of patients probably do not require hospitalisation; however, hospitalisation in even a small proportion of 
TABLE 5 Projected costs of 18-month course of guideline-recommended oral therapy for nontuberculous mycobacterial disease

Guideline/regimen
Total costs in 2008 CAD

Drug Nondrug Total

Total costs USD

Drug $^{\#}$ Nondrug" Total

\author{
ATS/IDSA [1] \\ Daily azithromycin $(250 \mathrm{mg})$, rifampin $(600 \mathrm{mg})$ and ethambutol $(800 \mathrm{mg})$ \\ Daily clarithromycin (100 mg), rifampin $(600 \mathrm{mg})$ and ethambutol $(800 \mathrm{mg})$ \\ Three-times-weekly clarithromycin $(1000 \mathrm{mg})$, rifampin $(600 \mathrm{mg})$ and ethambutol $(1200 \mathrm{mg})$ \\ Three-times-weekly azithromycin $(500 \mathrm{mg})$, rifampin $(600 \mathrm{mg})$ and ethambutol $(1200 \mathrm{mg})$ \\ BTS [12] \\ Daily rifampin $(600 \mathrm{mg})$ and ethambutol $(800 \mathrm{mg})$ \\ Daily clarithromycin $(1000 \mathrm{mg})$, rifampin $(600 \mathrm{mg})$ and ethambutol $(800 \mathrm{mg})$ \\ Daily ciprofloxacin (1500 mg), rifampin $(600 \mathrm{mg})$ and ethambutol $(800 \mathrm{mg})$
}

$\begin{array}{llllll}3817.80 & 2010.50 & 5828.30 & 8942.40 & 2702.91 & 11645.31 \\ 4989.60 & 2010.50 & 7000.10 & 9622.80 & 2702.91 & 12325.71 \\ 2601.90 & 2010.50 & 4612.40 & 4953.60 & 2702.91 & 7656.51 \\ 2742.30 & 2010.50 & 4752.80 & 6479.28 & 2702.91 & 9182.19 \\ & & & & & \\ 2106.00 & 2010.50 & 4116.50 & 4525.20 & 2702.91 & 7228.11 \\ 4989.60 & 2010.50 & 7000.10 & 9622.80 & 2702.91 & 12325.71 \\ 3844.80 & 2010.50 & 5855.30 & 10832.40 & 2702.91 & 13535.31\end{array}$

Nondrug costs include once-monthly assessment of complete blood count and liver enzymes, two sputum specimens every 2 months, computerised tomography (CT) scan at beginning and end of therapy (otherwise chest radiography (CXR) every 3 months), physicians' and facility fees (Canadian costs differ from our primary analysis in that facility fees for physician visits and CXR have been removed to model treatment in a nonhospital setting and facilitate comparison with the Medicare reimbursement structure; facility fees for CT scans have been retained, since CT scanners are generally operated and maintained by hospitals in Ontario, Canada, financed from hospital global budgets). Because the choice of regimen will depend more upon tolerability and goals of therapy (cure versus suppression) and because we cannot adequately assign a relative toxicity or efficacy of the regimens (greater toxicity or less effective regimens may lead to additional costs), the table should not be used to make clinical decisions. ATS: American Thoracic Society; IDSA: Infectious Diseases Society of America; BTS: British Thoracic Society. * : US drug costs derived according to the methods of BALLARINO et al. [4] and from personal communication with the authors. ": sources of nondrug costs are Medicare reimbursement rates calculated as the median of national nonfacility limiting charges from the centre for Medicare and Medicaid services (for physician fees and imaging) and mid-point costs from the 2008 Medicare Clinical Laboratory Fee Schedule (microbiology and blood tests); Medicare reimbursement rates are generally lower than those of private insurers, so the costs may be substantially higher for patients in the USA whose care is being charged to private insurance.

patients could raise the average costs substantially. Notably, in our population, eight patients underwent surgery (surgical biopsy, lobectomy or pneumonectomy) as part of their assessment or treatment. In our total treatment cost measurements and our cost estimates for a single, uncomplicated 18month treatment course (table 5), we did not account for the high rates of treatment failure and disease recurrence that are known to occur [1] and undoubtedly contribute heavily to the overall economic burden of pulmonary NTM. However, we did find that monthly costs of oral-only therapy are not inconsistent with the commonly accepted costs of treating chronic diseases, where therapy is continued indefinitely. We suggest that it may be just as important to focus on monthly costs, because treatment is often recurrent and sometimes chronic. Finally, the modelling of treatment cost may have been limited by the absence of information regarding the extent of radiographic disease (we had only data regarding cavitation) and macrolide resistance (most of our patients' isolates were not tested for drug susceptibility).

The generalisability of our work to other healthcare systems may be questioned. We have made a limited comparison to the USA, with regard to drug costs only. The applicability of comparisons between the USA and Canada is not clear, given the differences in medical cost-payers (society as a whole versus individuals). Regardless, we have expanded the comparison between the costs of treatment in Ontario, with those of treatment in the USA in table 5, wherein the cost of a projected uncomplicated 18-month treatment course for pulmonary MAC is presented. The modelled cost in the USA was 1.7-2.3-fold greater than the cost in Ontario, a difference driven largely by medication costs, which comprise the bulk of treatment costs and were expected to be 1.9-2.4-fold greater in the USA. The difference between the USA and Ontario may be even greater than we modelled, since we used Medicare reimbursement rates for nondrug costs, which are lower than reimbursed rates from private insurers: a study in 1993 estimated that Medicare reimbursement was $76 \%$ that of private insurance carriers [13]. Even though our model is probably a gross simplification, it appears to be clear that the cost of treating pulmonary NTM disease in the USA would be at least double the cost in Ontario.

The applicability of our work to systems where medical costs are generally borne by society is much greater. Our healthcare system in Ontario provides most prescription drugs free of charge to patients who are $\geqslant 65$ yrs of age or who are receiving social assistance. Furthermore, the provincial drug plan includes automatic substitution of generic drugs and our calculations utilised costs of generic drugs when available. 38 of our patients were $\geqslant 65 \mathrm{yrs}$ of age and several additional patients were receiving provincial drug benefits as part of their social income assistance. Finally, the costs of physicians' visits, hospital facility fees, home nursing visits, ambulatory parenteral drug therapy and diagnostic tests requested by a physician are borne completely by the Ontario Ministry of Health and Long Term Care. As a result, nearly half of our patients' treatment comprised exclusively societal costs, rather than costs borne by an individual or a private health insurance programme. In this context, we think that our costs reflect societal costs of the ambulatory medical management of pulmonary NTM disease very well. Based on the differing recommendations between the ATS [1] and the British Thoracic Society (BTS) [12] NTM guidelines regarding choice of specific 
drugs, we have presented in table 5 projected costs of an 18month course of therapy. It is evident that the first-line regimen from the BTS guidelines (rifampin and ethambutol) is by far the least expensive. However, because the choice of regimen usually depends more upon tolerability and goals of therapy (cure versus suppression) and because we cannot adequately assign relative toxicity or efficacy of these regimens (greater toxicity or less effective regimens may lead to additional costs), the values in table 5 should not be used to make clinical decisions.

The rising prevalence of pulmonary NTM infections will have an increasingly large impact on population health and heath expenditures. We observed that the cost of treating this disease is substantial, but not inconsistent with the costs of wellestablished therapies for other chronic diseases. Furthermore, the cost varied greatly with the use of parenteral therapy and with the presence of $M$. abscessus infection. In light of our findings, we do not think that the cost of treating pulmonary NTM disease should discourage therapy. The identification of new, less expensive alternative therapies may be most helpful for $M$. abscessus infection and when intravenous antibiotics are deemed necessary.

\section{SUPPORT STATEMENT}

This work was supported in part by Nontuberculous Mycobacteria Information and Research (NTMir), Toronto, Ontario, Canada.

\section{STATEMENT OF INTEREST}

A statement of interest for T.K. Marras can be found at www.erj. ersjournals.com/site/misc/statements.xhtml

\section{REFERENCES}

1 Griffith DE, Aksamit T, Brown-Elliot BA, et al. An official ATS/ IDSA statement: diagnosis, treatment, and prevention of nontuberculous mycobacterial diseases. Am J Respir Crit Care Med 2007; 175: 367-416.
2 Huang JH, Kao PN, Adi V, et al. Mycobacterium avium-intracellulare pulmonary infection in HIV-negative patients without preexisting lung disease: diagnostic and management limitations. Chest 1999; 115: 1033-1040.

3 Marras TK, Chedore P, Ying AM, et al. Isolation prevalence of pulmonary non tuberculous mycobacteria in Ontario. Thorax 2007; 62: 661-666.

4 Ballarino GJ, Olivier KN, Claypool RJ, et al. Pulmonary nontuberculous mycobacterial infections: Antibiotic treatment and associated costs. Respir Med 2009; 103: 1448-1455.

5 American Thoracic Society. Diagnosis and treatment of disease caused by nontuberculous mycobacteria. Am J Respir Crit Care Med 1997; 156: S1-S19.

6 Burgos M, Gonzalez LC, Paz EA, et al. Treatment of multidrugresistant tuberculosis in San Francisco: an outpatient approach. Clin Infect Dis 2005; 40: 968-975.

7 Hutchinson AB, Farnham PG, Dean HD, et al. The economic burden of HIV in the United States in the era of highly active antiretroviral therapy. J Acquir Immune Defic Syndr 2006; 43: $451-457$.

8 American Diabetes Association. Economic costs of diabetes in the US in 2007. Diabetes Care 2008; 31: 596-615.

9 Stroupe KT, Teal EY, Weiner M, et al. Health care and medication costs and use among older adults with heart failure. Am J Med 2004; 116: 443-450.

10 Jenkins PA, Campbell A, Banks J, et al. Clarithromycin ws ciprofloxacin as adjuncts to rifampicin and ethambutol in treating opportunist mycobacterial lung diseases and an assessment of mycobacterium vaccae immunotherapy. Thorax 2008; 63: 627-634.

11 Kobashi Y, Matsushima T, Oka M. A double-blind randomized study of aminoglycoside infusion with combined therapy for pulmonary Mycobacterium avium complex disease. Respir Med 2007; 101: 130-138.

12 British Thoracic Society. Management of opportunist mycobacterial infections: Joint Tuberculosis Committee guidelines 1999. Thorax 2000; 55: 210-218.

13 Miller ME, Zuckerman S, Gates M. How do Medicare physician fees compare with private payers? Health Care Financ Rev 1993; 14: 25-39. 